\title{
Urologists and radiation oncologists: working together for patient care
}

\author{
Joseph Chin, MD, FRCSC
}

See related article on page 197

$\mathrm{T}$

he report by Pearce and colleagues ${ }^{1}$ on differences in management recommendations by urologists and radiation oncologists for clinically localized prostate cancer serves as an update on 2 previous reports on the subject, published respectively 1 and 2 decades earlier. ${ }^{1,2}$ It is apparent that more clinicians now use risk stratification, life expectancy and survival benefits in their management algorithms. The higher degree of concordance in management recommendations for low-risk disease reflects the improved understanding of the natural history and the concept of competing risks, ${ }^{2}$ as well as the concept of therapeutic equipoise with various options. The findings on the comfort level for both groups with "watchful waiting" will hopefully translate into successful accrual and completion of the START (Surveillance Therapy Against Radical Treatment) trial comparing active surveillance and selective delayed treatment versus immediate treatment.

As the authors alluded to, the differences in recommendations for moderate-risk disease are likely multifactorial. Remunerative and "turf protection" considerations should not be, but realistically are, contributing factors. The relative lack of familiarity with the other specialty may translate into reluctance to recommend treatment modalities other than what they deliver themselves. Urologists may not be as familiar with the latest technical developments and improvements in conformal or intensity-modulated radiotherapy. Conversely, radiation oncologists may be less informed about improvements in surgical outcomes of urinary continence and erectile function preservation. This dichotomy underscores the value of multidisciplinary care conferences (MCCs), which are now functional in most, if not all, academic and many community practice settings. For urologists practising in more remote communities, advances in electronic and telecommunications are facilitating participation in tumour boards and MCCs. ${ }^{3}$ More dialogue with colleagues in other disciplines should increase the comfort level in discussing and possibly recommending alternative forms of management for our patients.

The results of the survey pertaining to cryotherapy also illustrate the lack of familiarity with this modality among Canadian clinicians, as there is very limited availability and exposure to this technology in Canada, either as primary or salvage therapy. This lack of familiarity is apparent in the responses regarding side effects of cryotherapy. Only $50 \%$ of either group thought cryotherapy would likely cause significant erectile dysfunction. In fact, $80 \%-90 \%$ of patients have erectile dysfunction with complete prostate cryoablation. Similarly, the majority of both groups thought that urinary incontinence would occur in up to $20 \%$ of cryotherapy patients. In fact, the rate of urinary incontinence after primary cryoablation should be under $10 \%{ }^{4}$

Patients need to receive unbiased, balanced and complete information before making treatment decisions. Radiation oncologists and urologists need to be up-to-date on their own as well as related specialties to optimally provide patients with therapeutic options and recommendations.

\section{Competing interests: None declared.}

From the Division of Urology, London Health Sciences Centre, St. Joseph's Health Care London, University of Western Ontario, London, Ont.

\section{References}

1. Pearce A, Newcomb C, Husain S. Recommendations by Canadian urologists and radiation oncologists for the treatment of clinically localized prostate cancer. CUAJ 2008;2:197-203.

2. Albertsen PC, Hanley JA, Fine J. 20-year outcomes following conservative management of clinically localized prostate cancer. JAMA 2005;293:2095-101.

3. Bauman $\mathrm{G}$, Winquist $\mathrm{E}$, Chin JL. A pilot study of regional participation in a video-conferenced multidisciplinary genitourinary tumor board. Can J Urol 2005;12:2532-6

4. Long JP, Bahn D, Lee F, et al. Five-year retrospective, multi-institutional pooled analysis of cancer-related outcomes after cryosurgical ablation of the prostate. Urology 2001;57:518-23.

Correspondence: Dr. Joseph Chin; joseph.chin@|hsc.on.ca 